\title{
Uma Metodologia para Agrupamento e Extração de Informações de URLs de Phishing
}

\author{
Welton Augusto Rodrigues Santos ${ }^{1}$, Osvaldo Fonseca ${ }^{2}$, Elverton Fazzion ${ }^{1}$ \\ ${ }^{1}$ Universidade Federal de São João del-Rei $\quad{ }^{2}$ Universidade Federal de Minas Gerais
}

\begin{abstract}
Resumo. Apesar de avanços em mecanismos de prevenção e mitigação, o phishing continua uma ameaça. Uma das razões disso é que o agente responsável pelo envio dessas mensagens, o phisher, aprimora suas técnicas continuamente. Neste trabalho estudamos e caracterizamos um destes aprimoramentos: o uso pelos phishers de cadeias de redirecionamentos para ludibriar mecanismos de identificação e evitar bloqueio da infraestrutura de hospedagem do conteúdo malicioso. Propomos um método para agrupamento de mensagens e URLs em campanhas de phishing, e desenvolvemos um arcabouço para identificação da infraestrutura de hospedagem. Nossos resultados mostram que a infraestrutura de hospedagem das campanhas identificadas se concentra em provedores de computação em nuvem, mas que algumas campanhas são hospedadas por dispositivos em provedores de rede, possivelmente em dispositivos infectados. Isso indica diferentes abordagens de phishers, sugerindo esforços em diferentes frentes de combate.
\end{abstract}

\section{Introdução}

Apesar dos avanços alcançados no combate ao phishing, esses ataques ainda são muito comuns. As técnicas empregadas nos e-mails de phishing evoluíram e, atualmente, apresentam um alto nível de personalização alcançado através de engenharia social e dados comprados ilegalmente de ciber-criminosos [Las-Casas et al. 2016]. Durante a última Copa do Mundo, por exemplo, phishers enviaram e-mails oferecendo a venda de ingressos para convidados, comprando domínios com nomes nomes similares a "worldcup2018" de forma a convencer as vítimas da legitimidade da mensagem. Devido a esses e outros aprimoramentos, é necessário o acompanhamento constante para capturar estratégias atualizadas, o que permite sofisticar as técnicas de combate a essa atividade criminosa que gera bilhões de dólares de prejuízo para os usuários e organizações [Las-Casas et al. 2016].

Para enviar mensagens, o phisher utiliza uma complexa infraestrutura composta por máquinas zumbis (pertencentes a botnet) e servidores SMTP vulneráveis. Essa infraestrutura permite ao phisher ludibriar filtros baseados em blacklists, uma vez que máquinas legítimas são responsáveis pela disseminação de mensagens, e dificultar a tomada de ações legais contra eles, pois a identidade dos criminosos na rede é substituída pela infraestrutura legítima utilizada. Além disso, o phisher também pode utilizar dessa infraestrutura para hospedar o conteúdo presente nas mensagens de phishing como, por exemplo, páginas Web associadas a URLs presentes nas mensagens que simulam serviços legítimos para furtar informações.

Complementar ao processo de filtragem de mensagens, o rápido bloqueio de páginas de phishing torna os e-mails que as contém inócuos, uma vez que a possível vítima não conseguirá acessar o conteúdo malicioso. Ferramentas automatizadas para 
analisar classificar URLs em URLs legítimas e URLs de phishing são fundamentais para permitir combate efetivo ao phishing dada a complexa cadeia de redirecionamento utilizada para ofuscar a infraestrutura de hospedagem.

Nesse trabalho, propomos uma metodologia de análise para URLs presentes em mensagens de phishing, de forma a identificar e agrupar páginas maliciosas similares, facilitando o processo de análise por parte de operadores de rede. Mais especificamente, nossa metodologia calcula a similaridade entre cada par de páginas combinando informações de termos frequentes utilizados por phishers (e.g., please, fill) presentes no código fonte da página. A partir do cálculo de similaridades, a metodologia gera um grafo cujos componentes revelam os grupos de URLs com páginas similares e que, possivelmente, pertencem a uma mesma campanha de phishing.

Nós aplicamos nossa metodologia a um conjunto de dados coletados por honeypots de baixa interatividade distribuídos na Internet. Combinamos as informações dos componentes gerados com informações de rede, como os endereços IP de servidores acessados na porta 80 e capturados durante o acesso a uma página, e mostramos que existe (i) uma concentração de URLs similares em poucos provedores de infraestrutura, indicando que alguns phishers podem estar fazendo uso de cartões fraudados para a compra de computação em nuvem para hospedagem das páginas e (ii) componentes com uma complexa cadeia de redirecionamento que dificulta a identificação da hospedagem e, consequentemente, do phisher. Para ambos os cenários, apresentamos uma caracterização completa das URLs presentes nos componentes e realizamos estudos de casos de forma a entender o comportamento atual de phishers.

\section{Processamento de URLs de phishing}

Para este trabalho, realizamos a coleta de e-mails com origem maliciosa usando honeypots de baixa interatividade (seção 2). Em seguida, analisamos esses e-mails e identificamos as mensagens de phishing utilizando técnicas estado-da-arte (seção 2.2). Para cada mensagem identificada como phishing, utilizamos uma sequência de heurísticas estáticas para identificar URLs com conteúdo malicioso (seção 2.3). Por fim, acessamos essas URLs de forma a capturar informações sobre as conexões realizadas (redirecionamentos) e obter o conteúdo da página (seção 2.4). A Figura 1 sumariza o processo descrito nesta seção.

\subsection{Coleta de mensagens}

As mensagens foram capturadas por 12 honeypots de baixa interatividade instalados em diferentes países, sendo dois no Brasil, Estados Unidos e Holanda, e um na Argentina, Áustria, Austrália, Equador, Japão, Taiwan e Uruguai. Os honeypots são configurados para emular servidores proxy (HTTP e SOCKS) e relay (SMTP) abertos e simulam vulnerabilidades [Steding-Jessen et al. 2008]. Quando um agente malicioso se conecta ao servidor SMTP de um honeypot, ele é levado a crer que está interagindo com um servidor SMTP real operando como um relay aberto. O mesmo acontece com os protocolos HTTP/SOCKS, onde o agente é levado a crer que é capaz de estabelecer conexões com outros servidores SMTP na rede. Os honeypots utilizados nessa pesquisa não prestam serviço para nenhuma rede e não são anunciados publicamente. Dessa forma, assumimos que todas as mensagens recebidas por eles provém de spammers ou phishers. Toda a interação com os honeypots é registrada e as mensagens são armazenadas localmente 


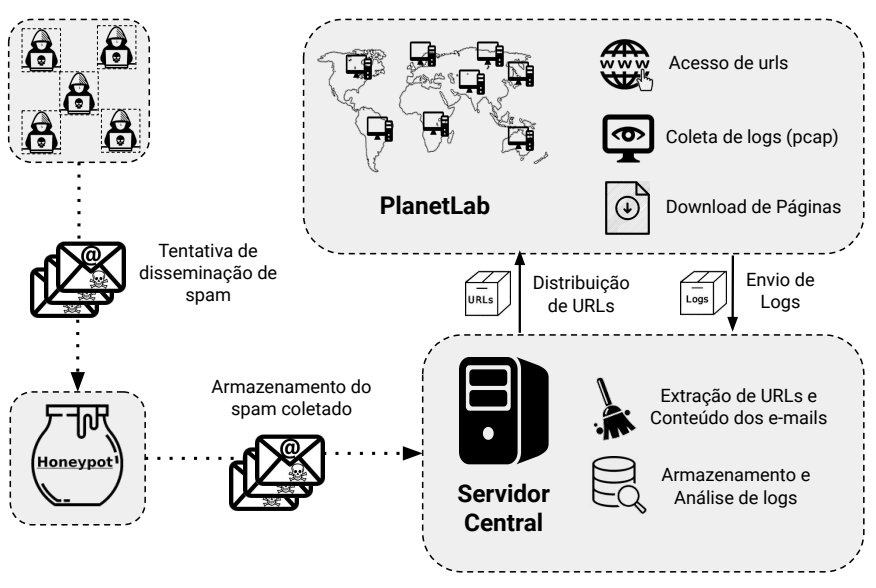

Figura 1. Metodologia de obtenção e acesso de URLs de phishing.

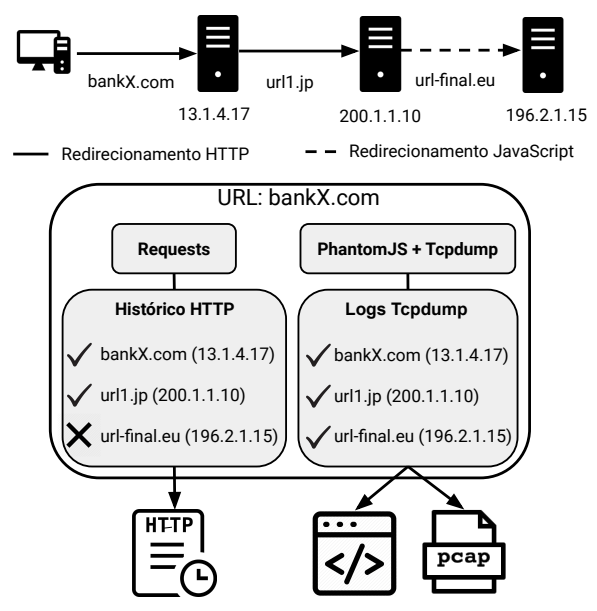

Figura 2. Página phisher escondida atrás de redirecionamentos

e enviadas para os servidores centrais do projeto diariamente. Nossos honeypots nunca encaminham as mensagens recebidas, com exceção daquelas mensagens cujos conteúdos indicam, de acordo com regras pré-definidas, que são mensagens de teste utilizadas pelo agente malicioso para verificar se proxies e relays abertos estão funcionando. Neste trabalho, analisamos todas as mensagens coletadas entre 29 de Abril e 14 de Maio de 2019.

\subsection{Identificação de mensagens de phishing}

Para separar mensagens de spam e phishing, utilizamos o classificador Naive Bayes. No nosso contexto, o classificador calcula e atribui a cada mensagem uma probabilidade de ser spam ou phishing, rotulando a mensagem com a classe com maior probabilidade. Para criar o treino do algoritmo, agrupamos as mensagens no idioma em inglês em campanhas [Calais et al. 2008], recuperamos uma mensagem representante de cada campanha, amostramos quinhentas mensagens aleatoriamente e realizamos uma classificação manual sobre a amostra. Ainda, cada uma das mensagens é submetida a um conjunto de regras para reduzir ruídos e alcançar uma maior padronização, evitando que um simples detalhe, como a diferenciação entre letras maiúsculas e minúsculas, afetem o modelo. Mais especificamente, convertemos cada caractere da mensagem para sua representação minúscula e removemos links, números, pontuações e stopwords. A composição final do conjunto de treino foram 230 mensagens de phishing e 270 mensagens de spam. Verificamos que a taxa de acerto da técnica de classificação foi de $92 \%$.

\subsection{Extração de URLs de phishing}

Em geral, as mensagens de phishing contém tanto URLs apontando para páginas falsas quanto URLs apontando para páginas verdadeiras (uma forma de aumentar a "legitimidade" da mensagem). Em algumas, por exemplo, a URL exibida para vítima é diferente daquela contida no HREF; a vítima lê e reconhece o link como referente a uma página legítima mas é redirecionada à página do atacante. Dessa forma, utilizamos heurísticas propostas na literatura para identificar URLs de páginas de phishing [Khonji et al. 2012]. Para cada URL verificamos se em seu corpo continha alguma das seguintes características: (i) urlatchar, presença de @ utilizado para associação com usernames a páginas falsas, (ii) urlbalink, termos relacionados a phishing (e.g. click, login, security), (iii) 
urlip, presença de endereço IP no domínio, (iv) urlnumport, alteração da porta WEB padrão 80 para portas altas e (v) urlstwodomain, presença de múltiplos subdomínios (e.g. http://empresa.com.phish.com). Caso a URL contenha alguma dessas características, ela será mantida no grupo de estudo. De todas as 621.531 URLs (469.763 únicas), apenas 119.122 URLs foram classificadas como phishing (89.619 únicas).

\subsection{Acessando URLs de phishing}

Para mascarar sua identidade, o phisher utiliza diversas facetas, dentre elas o acesso às páginas de phishing através de cadeias de redirecionamentos. Redirecionamentos são realizados a partir de requisições HTTP ou código JavaScript e uma cadeia de redirecionamento com diversos saltos pode utilizar as duas tecnologias.

O acesso a uma URL é realizado através de duas abordagens. Na primeira abordagem, a URL é resolvida a partir da biblioteca Requests, capaz de resolver somente redirecionamentos de requisições HTTP. Após o acesso com a biblioteca Requests, o histórico de requisições é salvo assim como o endereço IP e URL obtidos. Essa primeira abordagem consiste em uma estratégia rápida e de baixo custo computacional, porém não é completa, sendo incapaz de tratar URLs com redirecionamentos em JavaScript. A Figura 2 exemplifica a cadeia de redirecionamento da URL bankX.com que engloba tanto redirecionamento HTTP quanto redirecionamento via JavaScript. Dessa forma, utilizando apenas a biblioteca Requests não somos capazes de identificar a URL final.

Como visto na Figura 2, a biblioteca Requests retornaria o IP e URL obtidos no segundo salto e não atingiria a página final, do nosso interesse. Para lidar com situações similares, na segunda abordagem utilizamos o motor PHANTOMJS (https://github.com/ariya/phantomjs/) que consiste em um navegador para testes de aplicações web, sem interface gráfica, capaz de interpretar código JAVASCRIPT e que permite resolver URLs com perfil similar ao cenário mostrado na Figura 2. Quando o PHANTOMJS consegue acessar a URL, coletamos o endereço IP final, URL final e código fonte da página. Para registrar os endereços intermediários na cadeia de redirecionamento, utilizamos o software TCPDUMP, ferramenta de monitoramento e captura de pacotes. A partir do monitoramento da interface de rede do host, coletamos os endereços IP referentes a cada salto da cadeia de redirecionamento, como mostrado na Figura 2.

O acesso a URLs em maior escala é realizado utilizando a infraestrutura distribuída pertencente ao PlanetLab (https://www.planet-lab.org/). Dessa forma, os acessos às URLs são realizados de diversas regiões do mundo, ideal para o nosso trabalho, uma vez que grandes volumes de acessos de uma fonte centralizada poderiam despertar a atenção de phishers, comprometendo o sigilo dos monitoramento. É importante mencionar que não acessamos nenhuma URL a partir dos honeypots para o anonimato dessas máquinas.

A aplicação é estruturada sobre uma hierarquia mestre-escravo, conforme mostrado na Figura 1 onde um servidor central coordena vários outros servidores executando no Planet-LAB. Diariamente o servidor mestre envia aos servidores escravos um conjunto de arquivos com URLs. Visto que phishers mantém suas páginas ativas por curtos períodos de tempo e alteram constantemente os domínios onde residem suas páginas, os conjuntos de URLs enviados aos servidores passam por uma uma etapa de pré processamento. Nesta etapa um conjunto maior de URLs é triado de modo que reste ao final para distribuição somente URLs únicas e com ordem de prioridade precedida pela data de re- 
gistro mais recente. Dessa forma, tentativas de acesso à URLs repetidas ou expiradas são reduzidas, aumentando a margem de sucesso da aplicação. Em média, cerca de 33.600 URLs são processadas a cada dia, onde $48 \%$ são resolvidas com sucesso. Cada URL leva em média 12,75 segundos para ser processada com desvio padrão de 27,75 segundos.

\section{Agrupamento de Páginas}

O phisher, para tornar acessível o conteúdo de sua página maliciosa, associa uma ou mais URLs a uma página (ou conjunto de páginas similares). Dessa forma, cada URL associada a uma página pode ser correlacionada a outra URL através da proximidade existente entre os conteúdos de suas páginas, de tal forma que técnicas de agrupamento podem ser empregadas na identificação de campanhas de phishing.

\subsection{Detecção de páginas duplicadas}

Visto que várias URLs podem estar associadas a uma única página, submeter páginas iguais ao processo de extração de conteúdo e validação de idioma (apresentado na próxima seção), causaria maior consumo de recursos computacionais e tempo. Para evitar o processamento de páginas repetidas e identificar duplicatas foi construído um registro com os valores de hash MD5 de cada página. De forma que, antes de processarmos a página, verificamos se seu valor de hash consta no registro. Caso conste, a URL associada à página é associada ao hash já existente, caso contrário o novo hash é inserido na base associado à URL. Desta maneira conseguimos reduzir o conjunto total de páginas a processar de 119.122 para 43.028, 36\% da quantidade coletada.

\subsection{Extração de termos relevantes}

Neste trabalho optamos por lidar apenas com páginas com conteúdo em inglês, porém a técnica pode ser estendida a outros idiomas. Ao iniciar o processamento de uma página, todo o seu texto é extraído e determinamos o seu idioma com a biblioteca Langdetect (https://pypi.org/project/langdetect/), caso a página tenha sido escrita em idioma inglês, removemos pontuação e stopwords de seu corpo e avançamos para as próximas etapas do processo. Em seguida, duplicamos o conteúdo textual, onde a primeira cópia é submetida a um filtro a partir de um dicionário, como descrito na seção 3.2.1, e a segunda cópia a outro filtro destinado à identificação de nomes próprios, como descrito na seção 3.2.2.

\subsubsection{Filtro com dicionário}

Nesta etapa apresentamos duas alternativas desenvolvidas para extração de termos relevantes das páginas e discutimos a escolha para o trabalho.

Na primeira abordagem foi utilizado um dicionário do idioma inglês para identificar e preservar somente palavras pertencentes ao idioma. Porém, devido à abrangência do dicionário, quantidade significativa de palavras não possuíam relevância (como you, many, page, social). Outro problema consiste na frequente má estruturação de código presente nas páginas. Devido ao fato de muitas páginas conterem falhas em seu código, nestes casos, não foi possível distinguir com total precisão palavras reservadas de linguagens como CSS e JavaScript (e.g., hover, opacity, serif e display) e conteúdo textual pertencente ao idioma inglês prejudicando a eficiência do parser utilizado. Dessa forma, optamos por utilizar a segunda abordagem, descrita a seguir. 
Na segunda abordagem para a extração de termos, utilizamos um dicionário de termos associados a phishing baseado no trabalho de [Las-Casas et al. 2016]. Para atualizar as categorias presentes em [Las-Casas et al. 2016], executamos o modelo word $2 \mathrm{vec}^{1}$ para a base recente e identificamos uma nova categoria relacionada a conteúdo adulto, resultando nas seguintes categorias de termos relacionados a phishing: Tratamento, Menção a Dinheiro, Pedido de Resposta, Urgência, Formulário, Segurança e Conteúdo Adulto. Esta técnica também reduziu a adição de termos irrelevantes como o ocorrido na primeira abordagem, sendo então a técnica adotada.

\subsubsection{Extração de nomes}

Para obter maior acurácia na identificação de páginas com conteúdo similar, empregamos um mecanismo para identificar nomes próprios, uma vez que a extração de nomes de entidades permite identificar com maior precisão ataques contra instituições específicas. Para detectar nomes utilizamos o modelo pré treinado $S p a c y^{2}$. Dessa forma, submetemos o texto da página no modelo e extraímos os nomes próprios identificados assim como suas respectivas frequências.

\subsection{Construção do grafo}

Nesta seção descrevemos a construção do grafo de similaridade de páginas. Cada vértice nesse grafo representa uma página e os pesos das arestas quantificam a similaridade entre as páginas conectadas. Os pesos são valores reais dentro do intervalo $[0,1]$, proporcionais à semelhança entre as páginas conectadas pela aresta e determinados a partir do coeficiente de Jaccard. O coeficiente de Jaccard mensura a proximidade entre dois objetos calculando a razão entre a quantidade de atributos em comum e a quantidade de atributos totais (união entre os atributos contidos nos dois objetos). Para obter o coeficiente de Jaccard entre duas páginas, utilizamos como atributos as palavras obtidas no pré processamento das páginas descrito na seção 3.2. Portanto, o valor de uma aresta $A_{i j}$ entre duas páginas $P_{i}$ e $P_{j}$ é a razão entre a quantidade de palavras que aparecem em ambas as páginas, $P_{i} \cap P_{j}$, e a quantidade de palavras que aparecem em qualquer uma das páginas, $P_{i} \cup P_{j}:\left(P_{i} \cap P_{j}\right) /\left(P_{i} \cup P_{j}\right)$.

\subsection{Identificação de campanhas}

Para identificar conjuntos de páginas com objetivo comum, primeiro removemos arestas cujo peso seja inferior a um limiar $\alpha$, tal que $0<\alpha \leq 1$ e, em seguida, extraímos os componentes conectados do grafo. Para diferentes valores de $\alpha$ obtemos diferentes quantidades de componentes. Da mesma forma também observamos a variação na densidade de páginas por componente, descrita na Figura 3, onde é exibida a razão entre a quantidade de páginas por componente para cada limiar. Quando $\alpha$ se aproxima de 0.1 , componentes maiores contem páginas pouco semelhantes e, opostamente, quando $\alpha$ se aproxima de 1.0 é gerado um número maior de componentes, mas cujas páginas são mais semelhantes entre si. Para escolher o valor de $\alpha$, buscamos pelo ponto de inflexão na curva do gráfico da Figura 3, o limiar para o qual aumentar o valor de $\alpha$

\footnotetext{
${ }^{1}$ https://radimrehurek.com/gensim/models/word2vec.html

${ }^{2}$ https://spacy.io/usage/linguistic-features\#section-named-entities
} 


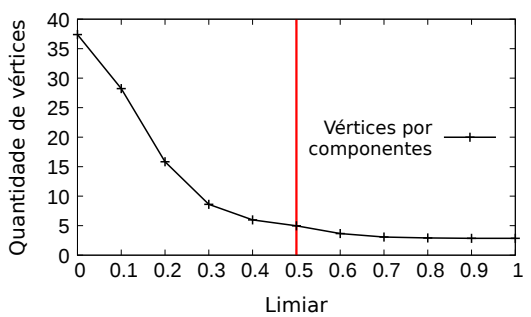

Figura 3. Média de vértices por componentes variando o limiar $\alpha$.

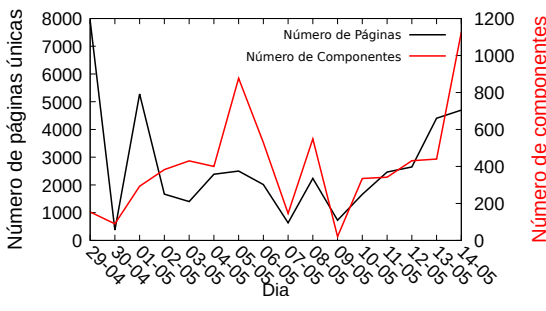

Figura 4. Distribuição do número de Páginas e Componentes observados por dia.

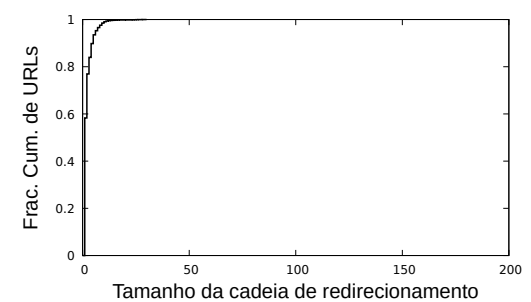

Figura 5. Tamanho das cadeias de redirecionamento para URLs.

têm pouco impacto no tamanho dos componentes. Considerando o gráfico da Figura 3, observamos que valores de $\alpha$ maiores que 0.5 não reduzem significativamente o tamanho dos componentes. Dessa forma, para os próximos resultados utilizamos $\alpha=0.5$.

\section{Resultados}

Nesta seção mostramos como a metodologia proposta auxilia operadores e analistas de rede a extraírem informações relevantes sobre conteúdos de phishing a partir de URLs coletadas. Inicialmente, caracterizamos as páginas Web obtidas através da aplicação do processo de coleta da seção 2 e mostramos como a metodologia da seção 3 reduz o número de páginas a serem analisadas (subseção 4.1). Em seguida, caracterizamos os componentes identificados e mostramos como podemos utilizá-los para sumarizar informações importantes sobre o conjunto de páginas como a concentração de páginas em redes de hospedagem de conteúdo (subseção 4.2).

\subsection{Caracterização de páginas e componentes}

O gráfico da Figura 4 mostra que o número de páginas únicas por dia (curva preta) varia ao longo do tempo, indicando variação do comportamento do phisher na propagação de campanhas de phishing. Apesar do número de páginas únicas observadas ser da ordem de milhares de URLs, nossa metodologia consegue agrupar páginas similares, reduzindo o número de páginas a serem avaliadas para algumas centenas, como mostrado no gráfico da Figura 4 (curva vermelha). Esse resultado mostra que, apesar de existirem milhares de páginas únicas, muitas delas possuem uma estrutura similar e podem estar associadas a uma única campanha disseminada pelo phisher na rede.

Nossa técnica também revela que um aumento no número de páginas únicas num dado dia não está necessariamente correlacionado com o aumento do número de campanhas de phishing naquele dia. Esse comportamento pode ser visto contrastando o número de páginas únicas em um dia com o número de componentes. Por exemplo, no dia 29/04 foram observadas cerca de 8.000 páginas únicas agrupadas em cerca de 200 campanhas de phishing. Porém, se observarmos o dia 14/05, verificamos cerca de 4.500 páginas únicas agrupadas em aproximadamente 1.100 campanhas. Além disso, esse resultado corrobora estudos na literatura que mostram que alguns phishers automatizam o processo de ataques de phishing, utilizando um modelo de página padrão que varia pouco entre diferentes tipos de ataques, conforme identificado pela técnica no dia 29/04.

Também avaliamos a sequência de Sistemas Autônomos (ASes) contactados a partir da URL para alcançar o conteúdo da página de phishing. Para cada URL, extraímos 


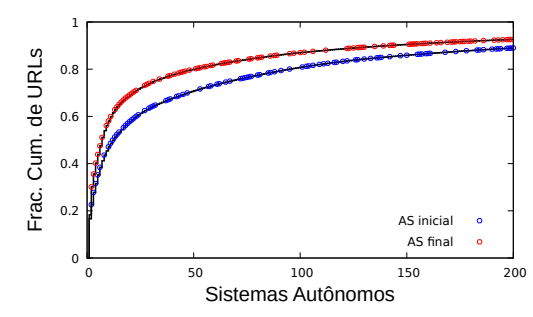

Figura 6. Distribuição de ASes iniciais e finais presentes nas cadeias de redirecionamento.

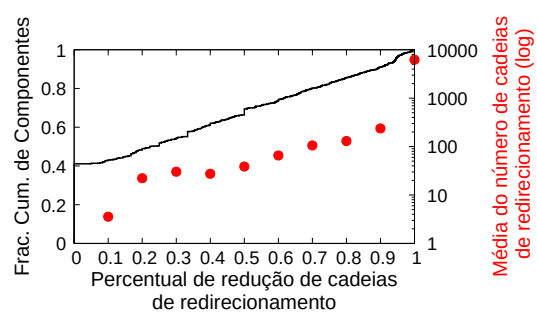

Figura 7. Agregação de cadeias de redirecionamento nos componentes.

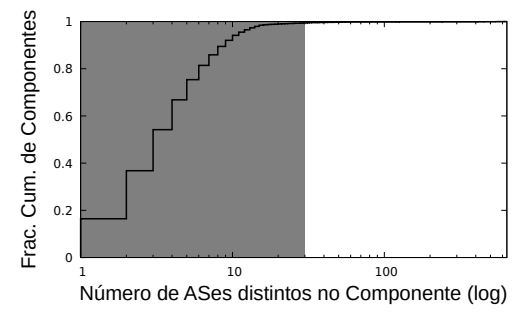

Figura 8. Distribuição de URLs únicas das cadeias de redirecionamento nos componentes.

a sequência $S_{i p}$ de endereços IP acessados na porta 80 em ordem cronológica no registro TCPDUMP daquela URL. Em seguida, para cada endereço IP na sequência $S_{i p}$, realizamos o mapeamento para o seu respectivo Sistema Autônomo utilizando a base do Team Cymru (http://www.team-cymru.org/), produzindo uma nova sequência $S_{a s}$ - endereços IP sem mapeamento não são considerados em $S_{a s}$. Por fim, substituímos ocorrências consecutivas de um mesmo AS $x$ em $S_{a s}$ por uma única ocorrência do AS $x$. Por exemplo, na sequência $\left\{A S_{1}, A S_{2}, A S_{2}, A S_{2}, A S_{3}, A S_{2}\right\}$ substituímos as três ocorrências do $A S_{2}$ na sequência por uma única ocorrência do $A S_{2}$, resultando em $\left\{A S_{1}, A S_{2}, A S_{3}, A S_{2}\right\}$. Chamamos a sequência final de $S_{a s}$ como cadeia de redirecionamento.

A Figura 5 mostra a distribuição acumulada do tamanho das cadeias de redirecionamento pelas URLs que conseguiram atingir uma página Web. Podemos observar que $41 \%$ das URLs analisadas utilizam algum tipo de redirecionamento que verificamos ser JavaScript (75\%) e HTTP (25\%). Além disso, como diferentes URLs podem apontar para uma mesma página, verificamos a distribuição da quantidade de cadeias de redirecionamento únicas em páginas com mais de uma URL.

Para melhor entender o tipo de sistema autônomo presente nessas cadeias, extraímos os ASes iniciais e finais de cada cadeia e consideramos o AS inicial igual ao AS final em cadeias com um único AS. Para identificar o tipo de negócio de cada AS, utilizamos a base de classificação de ASes da CAIDA (https://www.caida.org/data/asclassification/). A Figura 6 mostra a distribuição acumulada dos ASes iniciais e finais presentes nas cadeias de redirecionamento observadas. É possível notar que 50 ASes iniciais e finais estão presentes em $70 \%$ e $80 \%$ das cadeias de redirecionamento das URLs, respectivamente, e são, em sua maioria, ASes de conteúdo como indicado pelos círculos em cada curva da Figura 6. Esse resultado corrobora o fato que phishers podem estar fazendo uso de computação em nuvem para hospedar páginas de phishing utilizando cartões de créditos fraudados. Isso sugere que esforços nessas redes, como a instalação de algoritmos de identificação de páginas de phishing na rede e políticas mais rígidas na identificação do usuário que hospeda uma página, podem levar ao rápido bloqueio e a diminuição dos prejuízos causados por ataques de phishing.

\subsection{Análise dos Componentes}

Nesta seção avaliamos a infraestrutura de hospedagem dos componentes. A Figura 7 mostra o percentual de redução do número de cadeias de redirecionamento ao agrupar páginas similares, i.e., a redução do número de cadeias de redirecionamento a serem avaliadas quando consideramos os componentes $\left(1-\frac{\text { Cadeias unicas }}{\text { Total de cadeias }}\right)$. Podemos observar que, em $40 \%$ dos componentes, o agrupamento de páginas não reduziu o número de cadeias 
observadas, enquanto uma redução superior a $50 \%$ foi observada em $30 \%$ dos componentes. Para entender a eficácia das reduções observadas, analisamos a média do número de cadeias de redirecionamento nos componentes do intervalo $[x-0.1, x]$. Representamos essa média com um círculo em $x$ na Figura 7. Observamos que os maiores percentuais de redução $(x>0.6)$ ocorrem em componentes com cem ou mais cadeias de redirecionamento. Por exemplo, um componente com quase 10.000 cadeias de redirecionamento obtidas de suas páginas teve redução de quase $98 \%$, levando a poucas dezenas de cadeias de redirecionamento. Isso mostra a eficácia da metodologia proposta neste artigo para agrupar URLs similares.

Na Figura 8 verificamos o número de ASes distintos nos componentes provenientes das cadeias de redirecionamento de suas páginas. Verificamos que cerca de $95 \%$ dos componentes possuem 10 ASes ou menos relacionados à infraestrutura de redirecionamento e hospedagem das páginas. Além disso, observamos o tipo de negócio dos ASes em cada componente. Verificamos que componentes com poucos ASes possuem, em sua maioria, ASes de conteúdo (área sombreada na Figura 8). Isso reforça novamente a hipótese que phishers podem estar fazendo uso de computação em nuvem para hospedagem das páginas utilizando cartões fraudados. Para componentes com grandes quantidades de ASes (aŕea não sombreada com $x>30$ ), verificamos que o tipo de negócio da maioria dos ASes é provedor de infraestrutura. Neste caso, existe a possibilidade do phisher estar utilizando máquinas vulneráveis de usuários para hospedar o conteúdo de phishing, o que explica a grande diversidade de ASes relacionados a páginas similares.

\section{Trabalhos Relacionados}

Os trabalhos de agrupamento de e-mails maliciosos mais próximos à nossa proposta são (i) Li \& Hsieh [Li and Hsieh 2006], que agrupa mensagens utilizando o endereço IP dos links presentes nas mensagens, (ii) Fazzion et al. [Fazzion et al. 2014], que correlaciona atributos de rede e de conteúdo das mensagens para identificar infraestruturas de envio pertencentes ao mesmo phisher e (iii) Shoeb et al. [Shoeb et al. 2015], que identifica campanhas de spam utilizando o endereço IP final da URL presente nas mensagens. Nosso trabalho se diferencia desses trabalhos pois foca na identificação de campanhas de phishing através da análise do conteúdo de URLs dessas campanhas. Além disso, estendemos o entendimento da infraestrutura de hospedagem utilizada pelo phisher, possibilitando que analistas e operadores de rede possam identificar a infraestrutura de hospedagem subjacente e reduzir os prejuízos gerados.

\section{Artefatos resultantes}

Esse trabalho foi realizado em parceria com o CERT.br (Cristine Hoepers, Klaus StedingJessen e Marcelo Chaves) e a UFMG (Dorgival Guedes, Ítalo Cunha e Wagner Meira Jr.) como parte do projeto Honeynet (https://www.honeynet.org/) cujo objetivo é a constante investigação de ataques na Internet para o desenvolvimento e aprimoramento de ferramentas de combate. Em particular, nosso objetivo neste trabalho é a extração eficaz de informações para a identificação da infraestrutura de hospedagem utilizada pelo phisher. Dessa forma, medidas podem ser tomadas para que máquinas com o conteúdo malicioso de phishing sejam bloqueadas mais rapidamente.

A metodologia e resultados apresentados neste trabalho foram integralmente desenvolvidos pelo estudante e publicados no XIX Simpósio Brasileiro de Segurança da 
Informação e de Sistemas Computacionais [Santos et al. 2019]. Além disso, o estudante tem trabalhado em um sistema de alerta online para o CERT.br, utilizando as técnicas aqui apresentadas. Por motivos de sigilo de informações coletadas, o sistema desenvolvido não pode ser aberto ao público para não comprometer a identidade dos honeypots.

\section{Conclusão}

Neste trabalho apresentamos uma metodologia de agrupamento e análise de URLs presentes em e-mails de phishing. Mostramos como extraímos URLs maliciosas e identificamos o conteúdo das páginas apontadas pelas URLs, mesmo que o acesso esteja ofuscado por cadeias de redirecionamento. Para identificar campanhas de phishing, propomos um método baseado em grafos que combina informações do conteúdo com palavras tipicamente utilizadas em mensagens de phishing para calcular a similaridade entre as páginas identificadas. Nossas técnicas mostram que a infraestrutura de campanhas de phishing se concentra em redes de conteúdo, indicando que políticas mais rígidas de hospedagem de conteúdo podem contribuir no combate ao phishing. Além disso, mostramos que, em menor frequência, existem phishers que podem estar utilizando máquinas vulneráveis de usuários legítimos para a hospedagem de conteúdo malicioso.

\section{Referências}

Calais, P., Pires, D. E., Neto, D. O. G., Meira Jr, W., Hoepers, C., and Steding-Jessen, K. (2008). A campaign-based characterization of spamming strategies. In Proc. of Conference on Email and Anti-Spam (CEAS).

Fazzion, E., Las-Casas, P. H., Fonseca, O., Guedes, D., Meira Jr, W., Hoepers, C., Steding-Jessen, K., and Chaves, M. (2014). Spambands: uma metodologia para identificaçao de fontes de spam agindo de forma orquestrada. In Proc. of Brazilian Symposium on Information and Computational Systems Security (SBSeg).

Khonji, M., Iraqi, Y., and Jones, A. (2012). Enhancing phishing e-mail classifiers: A lexical url analysis approach. International Journal for Information Security.

Las-Casas, P. H., Fonseca, O., Fazzion, E., Hoepers, C., Steding-Jessen, K., Chaves, M., Cunha, Í., Meira Jr, W., and Guedes, D. (2016). Uma metodologia para identificação adaptativa e caracterização de phishing. In Proc. of Brazilian Symposium on Computer Networks and Distributed Systems (SBRC).

Li, F. and Hsieh, M.-H. (2006). An empirical study of clustering behavior of spammers and group-based anti-spam strategies. In Proc. of Conference on Email and Anti-Spam (CEAS).

Santos, W., Fazzion, E., Fonseca, O., Cunha, Í., Chaves, M. H., Hoepers, C., StedingJessen, K., Guedes, D., and Meira Jr, W. (2019). Uma metodologia para agrupamento e extração de informações de urls de phishing. In Proc. of Brazilian Symposium on Information and Computational Systems Security (SBSeg).

Shoeb, A. A. M., Mukhopadhyay, D., Al Noor, S., Sprague, A., and Warner, G. (2015). Spam campaign cluster detection using redirected urls and randomized sub-domains. In Social Informatics (Harvard).

Steding-Jessen, K., Vijaykumar, N., and Montes, A. (2008). Using low-interaction honeypots to study the abuse of open proxies to send spam. INFOCOMP, 7(1). 